\title{
A new MATLAB Simulink Toolbox for Dynamic Building Simulation with B.I.M. and Hardware in the Loop compatibility
}

\author{
Dietmar Siegele ${ }^{1}$, Eleonora Leonardi ${ }^{1}$, Fabian Ochs ${ }^{1}$ \\ ${ }^{1}$ University of Innsbruck, Unit for Energy Efficient Building, 6020 Innsbruck, Austria
}

\begin{abstract}
A new tool carnotUIBK was developed for dynamic building simulations within MATLAB. It consists of two parts: the first part is a MATLAB based object-oriented building model framework for handling data within building simulation matters, including import (of gbXML (B.I.M.), Excel and PHPP), export, data preparation and data analysis. The second is a simulation framework within Simulink, which provides the simulation engine. One of the main features of the new model is that it is tailored for hardware in the loop applications, controller development and indoor air quality simulations in terms of multi-zone modelling.
\end{abstract}

\section{Introduction}

A large number of (non) commercial dynamic building simulations are available on the market (e.g. TRNSYS, IDA ICE, Energy+, Modelica etc., see e.g. (Attia et al., 2012 or Crawley et al., 2008)). Most of these programs are for pre-design or design of buildings and HVAC to be used by architects or building engineers but are not (expect of Modelica) optimized for use in scientific practice, even if this is often claimed. Some models behave like a black-box (as the building model of TRYSYS), which is critical from the scientific point of view. First the reliability of such models cannot be proven by others and second the boundary conditions in which the models can be used accurately must be known. Furthermore, the numerical accuracy of building simulations is a problem not often discussed. It is hardly ever given in scientific papers (in contrast to measurements where the accuracy of the measurement equipment has always to be announced).

One specific application case within the scientific context where not all requirements can be fulfilled is Hardware in the Loop. Within Hardware in the Loop not only the building has to be simulated accurately but also the possibility to develop and simulate complex control systems must be available.

From the scientific point of view Modelica could be an alternative but there are some arguments against. First no comprehensive method is available to handle simulation data and to automatically create multi-zone models e.g. from a gbXML file. An idea of such a method in context with the library BuildingsSystems was presented by Nytsch-Geusen (2017), but the framework consists only of generators without a capability to analyse the results or even save complex models with different parameter variants. The capability for developing control systems within Modelica is compared to other tools quite small. In Majetta (2017) different controller types have been investigated with a quite high effort, which e.g. in Stateflow would had been an easy task. Also, Modelica delivers only possibilities to develop physical simulation models and not programs. For this reason no comprehensive graphical interfaces (GUI) or data analysis can be developed without knowing other programming languages (as e.g. Python).

MATLAB offers an object-orientated programming language and gives a capability to develop comprehensive GUIs and analysis. Simulink is a modelling framework which solves ordinary differential equations (in contrast to Modelica which solves physical equations). For Simulink a high number of so-called Toolboxes are available. They extend the basic functionality. The most important are a Toolbox for developing control systems (which is widely used e.g. in the automotive industry), Stateflow (for developing controllers based on stateflow charts) and an optimization Toolbox. Moreover, Simulink allows to choose different solvers (discrete and continuous with automatic time steps). The implemented error estimation functionalities ensure the high calculation accuracy needed for scientific purposes. One of the disadvantages of Simulink is that solving algebraic loops (so physical equations without time constants) is very slow. Also the direction of a model must be defined, so e.g. a medium in a pipe can only flows in one direction. Also Toolboxes for building and HVAC simulations are already available for MATLAB Simulink (e.g. CARNOT Toolbox (CARNOT 2018)). Other toolboxes (e.g. MLE+ (Bernal et al 2018) were developed but seem not to be further developed nor maintained. Graphical user interfaces (GUI) and clear structures to build reproducible complex models are missing in these toolboxes, such as e.g. in the CARNOT Toolbox. In the field of scientific building simulations, a large amount of data is necessary, and it needs to be well organised and managed.

For this reasons the carnotUIBK Toolbox (Siegele and Leonardi, 2019) has been developed within the project SaLüH (2018). By means of object-oriented programming a parameterized building model was developed, which is freely available to the community. It is fully compatible to the existing Toolbox CARNOT (CARNOT, 2018). The Toolbox requires MATLAB version R2018b or higher. 


\section{Functionality}

The tool carnotUIBK consists of two frameworks. A building model framework for handling data within building simulation matters and a building simulation model for the simulation itself.

The main functionalities of the building model framework within MATLAB are:

- Modern Graphical User Interface

- Object-oriented programming with the opportunity to use all methods directly (e.g. for batch simulation or parametric simulation studies).

- Data management of input data und results

- Creation of variants to simulate different variants

- Data import from gbXML, Excel and PHPP

- Standardized post-processing with pre-built functions with the possibility to extend without limitations

The simulation model in Simulink delivers the following main functions:

- Simulink library with all blocks and a basic HVAC library

- Templates for the Simulink model

- $\quad 1 *$ - and $2 *$-node models for the zone calculation

- UA, R-C and hygrothermal model for the opaque structures

- A Hardware in the Loop and co-simulation block which can be used in combination with BCVTB (Wetter 2010).

Moreover, examples for all functionalities are provided.

However, the MATLAB building model framework can also be used without Simulink. It is planned to support also other simulation engines as Modelica and Energy+.

\section{Workflow}

A building and HVAC simulation consists mostly of the following steps:

1. Definition of the geometry

2. Definition of the construction (structures)

3. Definition of gains and sources like people (power, moisture, $\mathrm{CO}_{2}$ ), electricity, moisture

4. Definition of the boundary conditions (weather, adjacent zones such as ground or neighbour)

5. Definition of the thermal zones

6. Creation of the building model

7. Definition of the HVAC systems

8. Creation of the HVAC systems

9. Simulation with a simulation engine

10. Post-processing (analysis) of the results

11. Validation of the simulation results

12. Parametric study or optimization by repeating all or several steps from 1 to 11 .

The steps 1 to 6 and the step 10 can be done in the GUI of the carnotUIBK Toolbox. The steps 7 and 8 (HVAC) and the step 9 (simulation) are proceeded in Simulink (the simulation engine). For the step 1 (geometry) and 5 (thermal zones) the gbXML standard can be used to import the necessary data.

\section{Methods}

\section{Object oriented Programming and Structure}

For programming the object-oriented language of MATLAB is used. There are many advantages by using this kind of programming style. Methods (which can be interpreted as functions in classical programming languages) are belonging to an object. This means e.g. the method 'plot' (which is also used by MATLAB itself) can be used to plot the geometry of the building, but also to plot a construction or a result. Only the object has to be known (the so-called caller). For this reason, method names can be short and simple and they don't have to be unique. Also parts of the framework (e.g. one sub-object) can be changed and updated without influencing other parts of the framework.

The building simulation framework uses one main object with different sub-objects (compare Figure 1). The main object is called 'BUILDING' and includes all necessary sub-objects and further global parameters. It also contains functions to start the simulation and to save the results of a simulation.

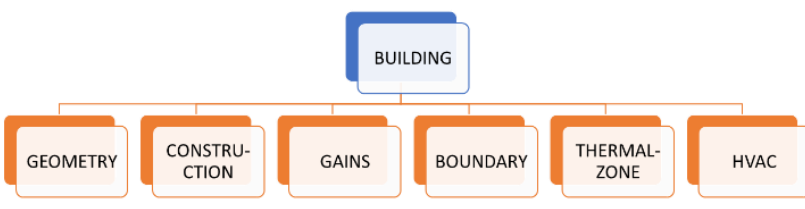

Figure 1: Scheme of the object structure in MATLAB.

The sub-object 'GEOMETRY' includes another subobject 'ROOM', which itself contains parameters of the room and another sub-object 'WALL'. Moreover 'GEOMETRY' provides methods to plot the room geometry or functions to check that only unique room names are used. The 'WALL' contains again parameters of itself (especially width and height, calculation model, sky-factor and boundary) and further sub-objects for windows and doors.

The sub-object 'CONSTRUCTION' contains the structures ('STRUCTURE') used within the simulation and all relevant parameters necessary for the calculation. It also contains methods to create the discretization for the models starting with a list of layers and their properties. Furthermore, again methods to plot (compare Figure 3) are provided.

'GAINS' stores the parameters for different kind of gains (compare section below) and again methods to plot them (compare also Figure 4).

The sub-object 'BOUNDARY' stores the information about the boundary conditions weather, ground and neighbour (each again a sub-object). They can be used in the 'GEOMETRY' as boundary. Methods to add the data in different ways (e.g. data import of weather files, e.g. generated with METEONORM ${ }^{\circledR}$ ) are provided.

In the sub-object 'THERMALZONE' the zones and their belonging intersections (connection between two zones) are defined in additional sub-objects. Further on 
parameters for the calculation model are defined there. Methods to plot the thermal zones are implemented.

The HVAC systems are defined in 'HVAC'. Systems can be defined (again as sub-objects) with parameters. The concept how to implement HVAC systems in the building is explained in the following sections below.

\section{Building model: Import, Export and GUI}

Thanks to object-oriented programming, a large number of import options can be implemented in a short time. Since one of the aims of the tool is his adaptability, the user can choose between many possibilities to import the data, depending on what suits best to his need or to the availability of the data. There are no limits to extend the existing import methods or to add new ones.

A gbXML (gbXML, 2017) import is implemented. gbXML is compatible to the IFC standard which is widely used in B.I.M. The import of a gbXML file can be used to import the geometry (including the assignment of the construction) and the thermal zones. The import of the structures and gains is right now not implemented, but they can be defined in the GUI. The gbXML standard do not include all kind of information that is necessary to produce an accurate building simulation. For this reason, in the GUI of carnotUIBK several additional parameters can be changed.

An import from the PHPP (Passive House Project Package) (Feist, 2018) is also available as a fast and efficient import option for 1-zone models.

To allow fast and efficient changes on multiple parameters an Excel import and export of all of the parameters (expect of HVAC) was realised.

Furthermore, a GUI was created. It is possible to use the GUI to go through most of the points of the workflow. The GUI can be started from any folder with the MATLAB command 'start_carnotUIBK'.

Figure 2 shows the interface to define the wall of one room.

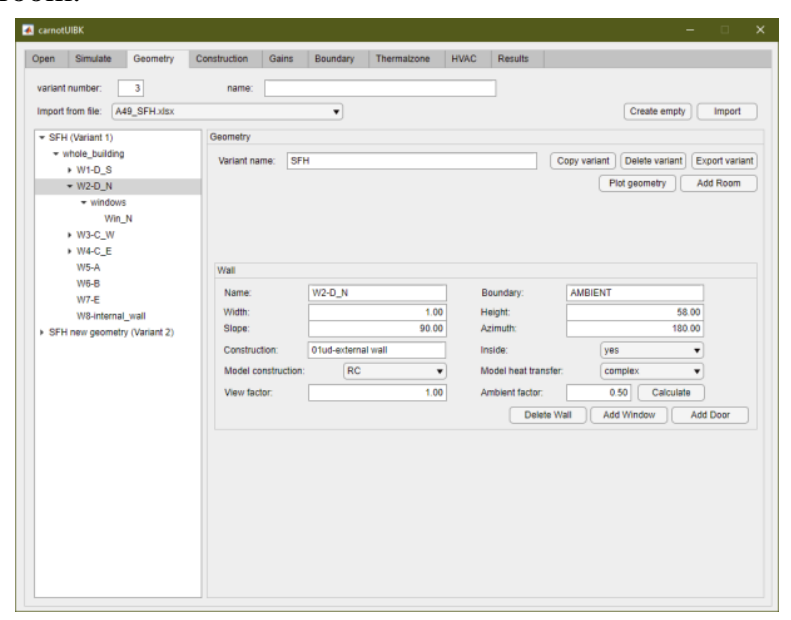

Figure 2: GUI of the geometry.

Figure 3 shows a structure in the GUI.



Figure 3: GUI of the construction.

Figure 4 shows how gains and sources can be defined within the interface.

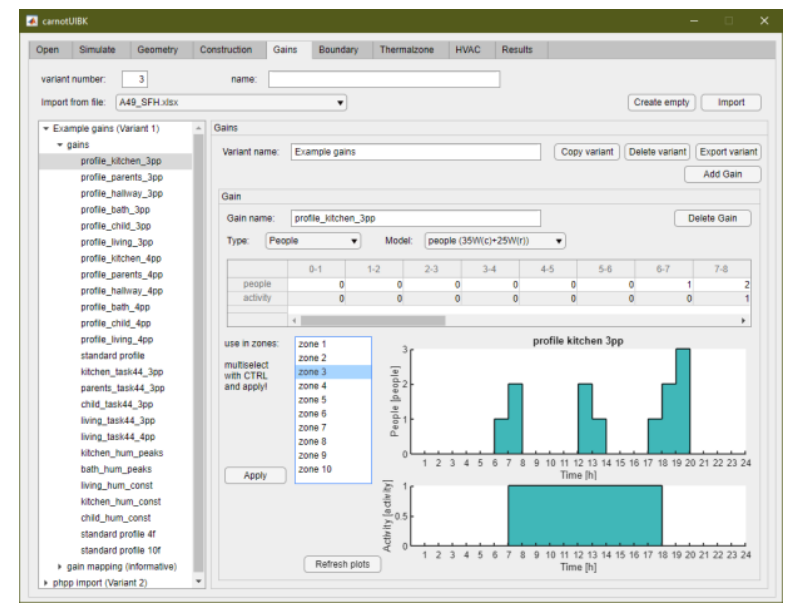

Figure 4: GUI of the gains and sources.

\section{Building model: Post-processing}

Some predefined functions are implemented in the model for the post processing. Temperature, humidity and $\mathrm{CO}_{2}$ graphs and data are available. Heating demand, cooling demand, balances of losses and gains can be plotted for every zone and month. Some useful plots as heat load sorted by ambient temperature and by hours, the temperature arranged by the hours are available. A comparison between the results of the simulation and of a PHPP can be done. The above-mentioned functions can be used out of the GUI or as usual MATLAB functions in user-defined scripts.

\section{Description of the used Models: Construction}

Different models for the opaque constructions are implemented and can be combined in a very flexible way depending on the purpose of simulation: Thermal Conductance (UA), the resistance-capacity (R-C, also called Beuken model) and a 1-D hygrothermal model (Siegele, 2014). 2D FD or FE models can be coupled, too but are not available in the standard version (Siegele, 2014).

For all models the heat transfer coefficients (convective and radiative) are calculated automatically during the 
simulation depending on the boundary and the orientation (Feist, 1994).

The UA model includes major simplifications and thus has the best computational performance. Only two temperatures are calculated (the external and the internal surface temperature). There is no capacity considered.

Figure 5 shows the R-C model scheme. This model is taken from the CARNOT Toolbox. Compare Feist (1994) for more details and the effect due to the simplifications. For this model the conductivity, the density and the specific heat capacity must be defined for each layer.

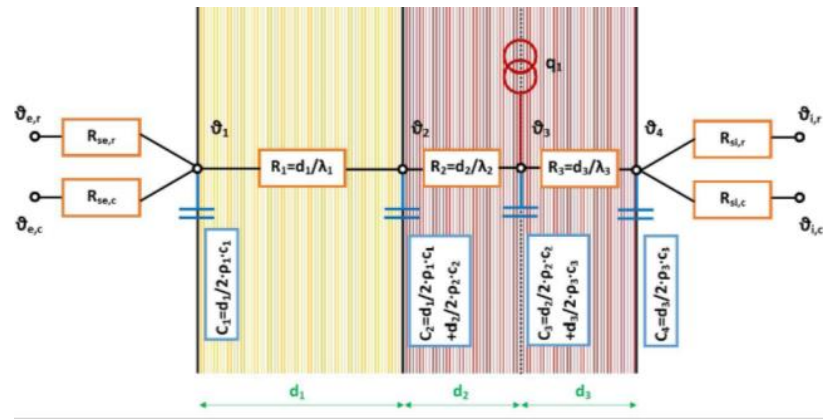

Figure 5: Scheme of the RC model of the structure.

If a detailed investigation of the indoor humidity is required (e.g. for determination of thermal comfort or to proof moisture safety), the hygrothermal model should be used. The hygrothermal model for Simulink was presented in Siegele (2014). The model uses differential equations for the coupled energy and the mass transfer. A quite high number of input information is required (hygrothermal properties and functions). Figure 6 shows a generic sorption isotherm, which must be known for each material. Discrete values are used to describe it (compare with 'Example of a Building Model').

With the R-C model and hygrothermal model it is also possible to include a source within the wall (for example for a floor heating system). These features are provided for expert users.

One advantage of this approach is that the calculation model can be chosen for each wall. This allows for e.g. a detailed simulation of one or some zones with a hygrothermal model and less important zones with a R-C model. This saves simulation time. Also, if a structure is defined once with all hygrothermal parameters it can be calculated with all other calculation models, which means also UA or RC, without the need of changing something or duplicate it. Moreover, this leads to a reduction of the risk to introduce mistakes by changing calculation models.

For the windows the existing model of the CARNOT library with a transfer function and an incident angle modified g-value is used. Improved models will be implemented in the near future.



Figure 6: Sorption isotherm for the hygrothermal model (Davis, 2004).

\section{Description of the used Models: Zone Model}

The zones can be calculated by means of two models: the one-star model and the two-star model.

In the so called 'one-star model', all powers are summed up in one node (compare Figure 7). The node's capacity represents the whole mass of the building (Davies, 2004;

Feist, 1994). This model is a big simplification that should lead to some inaccuracy in the results. This means the dynamic response of the temperature is not correct. But it can e.g. be used in thermal zones that are less relevant for the investigation.

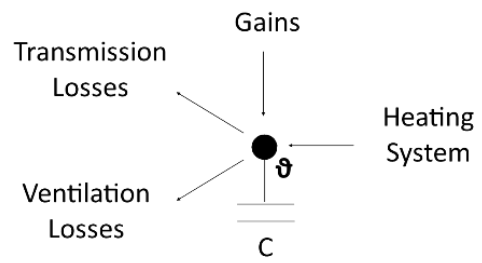

Figure 7: One-star model.

A most accurate and complex model, the "two-star model" or "complex model" is implemented. The behaviour of the building is described by means of two nodes: the radiative and the convective node (Davies, 2004; Feist, 1994). As shown in Figure 8 each surface is in exchange with each node. All powers are distributed to the convective and radiative node, depending on the type of gains and on the type of heating system. The surfaces (from the structure) and the convective node (the air mass) have a capacity, whereas the radiative node has only a small capacity for numerical reasons.

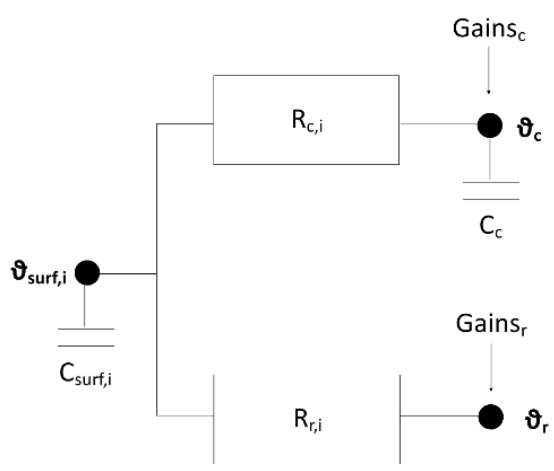

Figure 8: Two-star model. 


\section{Description of the used Models: Gains and Sources}

Different types of gains and sources can be defined and assigned to each zone:

- gains due to people,

- electricity,

- light and

- moisture source.

The internal gains due to people can be calculated in three ways. A presence profile and an activity profile have to be defined for the first two models. In the first model a constant value of $35 \mathrm{~W}$ (convective) and $25 \mathrm{~W}$ (radiative) per person is used. The moisture source and the $\mathrm{CO}_{2}$ emission are calculated based on the activity. The second model uses also for the emitted power the activity profile. For the third model the specific power profile $\left[\mathrm{W} / \mathrm{m}^{2}\right]$ has to be defined.

Light and other electricity (equipment) can be assigned in two ways: by a specific power profile $\left[\mathrm{W} / \mathrm{m}^{2}\right]$ or per zone [W].

A moisture source can be defined with a mass flow profile $[\mathrm{kg} / \mathrm{s}]$. The needed power for the evaporation is automatically calculated.

\section{Description of the used Models: Air Exchange}

In carnotUIBK three physical effects can be considered (compare Figure 9).

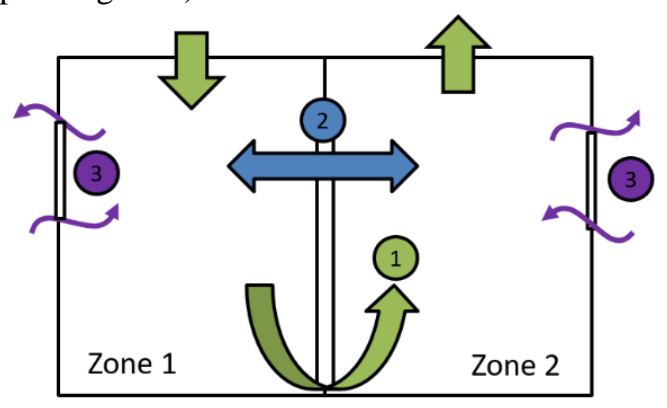

Figure 9: Infiltration and air exchange model.

Effect number 1 is the forced convection between the zones caused by a ventilation system. This effect has to be modelled with a HVAC system. Effect number 2 is the pressure driven air exchange between the zones that happens if a door is open. Effect number 3 is the infiltration and exfiltration to the ambient.

The air exchange between the rooms caused by open doors can be calculated in two different ways: by means of an empiric model or with a power law model. The empiric model was described in Rojas (2015) and is used as a standard model. It calculates the mass flow depending on the temperature and the humidity difference between two zones. The second model calculates the mass flow by means of a power law. The volume flow depends on the density of the air, the dimension of the door and the difference of temperature. This model is accessible to expert users.

The zone-wise infiltration can be calculated by using a constant infiltration rate or by a pressure depending infiltration rate. The parameters for the first model can be defined in the sub-object 'ROOM'. The second model calculates an infiltration mass flow depending on two effects, the chimney effect and the wind pressure. The model can be used in combination with a window. The model is still in a testing status and not officially available.

\section{HVAC in the Building Model}

For the building simulation community it is extremely important to be able to rely on models that were developed and validated earlier. Very often this is difficult e.g. if similar variable names in different models are used. A closed parametric variable space is necessary to avoid such problems and to be able to consequently exchange models within the scientific community or a workgroup.

For this reason, carnotUIBK allows to define 'systems'. (These are comparable to the approach of the 'Types' in TRNSYS). The system numbers must be unique. In an object 'system' a parameter set in form of a MATLAB structure is defined. This parameter set is a closed parametric variable space. Models in Simulink have access to variables by using the variable prefix 'hvac.' .

The HVAC model itself can be freely programmed in Simulink with blocks, since the investigation of an innovative HVAC is often the aim of a building simulation in the scientific community. Full compatibility with the CARNOT Toolbox (which contains blocks for hydraulics, storages, etc.) is ensured.

HVAC models itself can be easily exchanged with Simulink libraries. In a future release the support of FMU (Function Mock-up Units) is planned. The FMI (Function Mock-up Interface) allows to exchange physical models through different simulation platforms. A compatibility e.g. to Modelica could be easily realized with this method.

To create new HVAC systems in Simulink a template of the library can be copied and modified. Several system blocks for Simulink and their parameter sets are predefined in the carnotUIBK library.

The analysis of the HVAC systems is user-specific. In the Simulink model is a data logger available with an input bus for the HVAC systems. All HVAC systems can use this bus to save simulation results.

\section{Hardware in the Loop}

A Hardware in the Loop (HiL) connection is implemented with BCVTB (Wetter, 2010). The Simulink block, which is used by Wetter (2010) to manage the connection was extended so that also continuous time steps can still be used. This is very important to avoid a stand-alone compilation of the whole Simulink model. With this approach the same model can be used for the building simulation with or without coupling with HiL.

The block can also be used for any kind of co-simulation that is realized with BCVTB.

This part is still work in progress and not an official part of the Toolbox. On request, the authors will gladly provide a beta version.

\section{Validation of the Model}

The models used in carnotUIBK were validated in most cases (compare with the corresponding publications in the 
section above). A validation of overall simulation model of carnotUIBK in term of a comparison with other simulations tools has been done by Magni et al (2019). In term of the energy balance the results are within the range of the other simulation tools used.

\section{Example of a Building Model}

\section{Building Description}

Based on a building model created within the frame of the research project SaLüH! (Ochs et al, 2019) an example of the workflow of carnotUIBK is demonstrated. The building is a typical multi-story building in Central Europe. It has five floors and two symmetrical apartments per floor (compare Figure 10).

The tool is also used in further research projects. Here only basic results can be presented.

Different cases of heating systems were e.g. investigated with this model (Calabrese, 2018).

Further investigations with this model were done to evaluate the application of enthalpy exchangers compared to heat exchangers in Siegele (2018). A great advantage of carnotUIBK is the hygrothermal model, which allows such kind of investigations with a high accuracy and considering all relevant mechanisms.

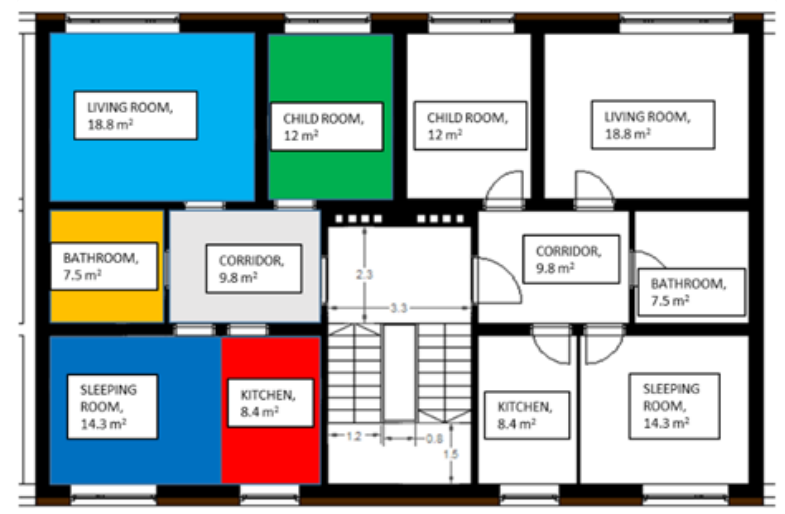

Figure 10: Ground plan of the building.

Within the project SaLüH! (Ochs et al, 2019) an air-to-air heat pump with secondary air was developed. With this building model the controller was developed. The controller allows a room-wise temperature control with an air heating system. This was only realizable with a high number of control states and a comprehensive simulation in Simulink.

Afterwards the controller was tested with Hardware in the Loop and a prototype of the heat pump. A publication with the results is in work.

\section{Definition of the Geometry}

The building was drawn in SketchUp (Trimble, 2018) by using the Openstudio Plugin (NREL et al, 2018). This plugin allows to generate a gbXML file. Many other products in the B.I.M. field allow to create such a file (e.g. Revit, archicad). All rooms are drawn with all relevant geometry elements (windows, doors). Constructions are assigned to all geometry parts (compare Figure 11, the different colours indicate different constructions).

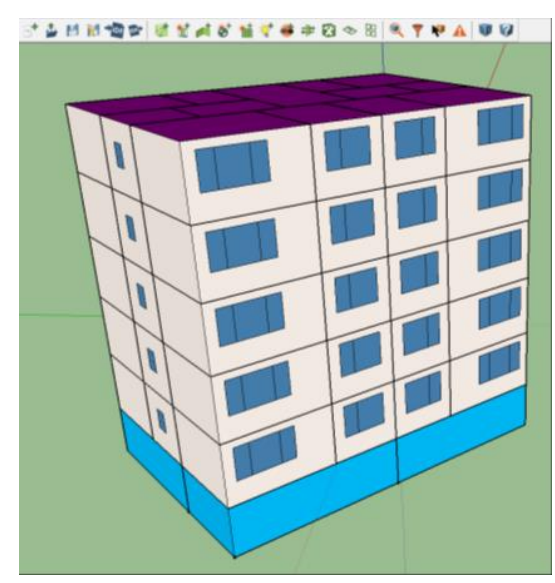

Figure 11: Building drawn in SketchUp and Openstudio.

Additional parameters can be set within the GUI, e.g. assigning a control variable to a window for opening or shading. In a next step, the calculation models can be chosen. In this case, the hygrothermal model was used for all investigations. All geometrical parameters, as orientation and so on are automatically defined out of the input file. Connections (intersections) between two rooms are automatically recognized and the boundary of each wall is generated.

\section{Definition of the Structure}

To define the structure the type of wished calculation model must be known. A structure can be simulated with an UA-model (steady-state behaviour), a R-C model for thermal simulations or a hygrothermal model for hygrothermal simulations. For the window the model with a transfer function is available.

Two ways are available to define the structure. With the GUI a R-C model of the wall can be defined. For hygrothermal models and for the definition of the windows the Excel import has to be used. A template can be found in the main folder. As the hygrothermal model requires a large amount of input data, it is necessary to use files for each material and the filename has to be specified. As data format the Delphin Material File Specification has been chosen. Example files are provided and the specification of the file format can be found in Vogelsang et al (2013).

In this example model all structures were defined with hygrothermal parameters and the simulation studies used different kind of combinations for the calculation models.

\section{Definition of the Gains and Sources}

The gains and sources can be defined in the GUI. Optional an import of the Excel using the template is possible. Persons, electrical devices, lights and additional moistures sources can be defined. For each gain a control input can be defined to control it (also with numerical values to scale output factors) during the simulation depending on time or variable states.

For the example building gains for persons, electrical devices and additional moisture sources were defined. Most of them are based on Dott et al (2013), some on internal documents. Different gains for different zone types were created and assigned to the zones. 


\section{Definition of the Boundary Conditions}

The boundary conditions can be imported in the GUI. Weather, ground temperature and neighbour temperatures profiles can be defined.

The weather data are imported from a file, in the case Innsbruck generated with Meteonorm ${ }^{\circledR}$ according the file specifications of CARNOT Toolbox.

For the ground and neighbour temperature profiles monthly values can be defined. The ground temperature has to be determined in the pre-processing e.g. according to the ISO 13370. In the template in one sheet this calculation is implemented, and the results can be taken as input data.

\section{Definition of the Thermal Zones}

The thermal zones can be defined in the gbXML file but also in the GUI. In this case a ten zone building model was realised. One flat (first floor, Northeast orientation) was used as a reference for the simulations. It was divided into six thermal zones. Below, above and adjacent flats, basement and staircase were each modelled as additional thermal zones.

Different calculation parameters for the models can be set and the calculation model itself $\left(1 *_{-}\right.$or $2 *$-node model) has to be defined. In order to reduce the simulation time in some cases only the reference flat was simulated. Also this can be realized by changing the calculation model to a profile. No additional changes have to be done.

\section{Creation of the Building Model}

By loading a template of a Simulink model the building model is already created. The only things which are left to do in Simulink are to add HVAC systems and connect them to the building. Moreover, it is necessary to connect controllers and control signals to the building e.g. if a strategy for window ventilation or shading should be considered.

In this example separate HVAC systems were used to control the window shading and opening and also to control the door opening position of doors between different zones.

\section{Definition and Creation of the HVAC Systems}

The HVAC systems are always a combination of a Simulink model and the corresponding parameter set. In the GUI systems can be imported and the parameters can be checked. A change of the parameters can be done within the MATLAB workspace.

Within the project SaLüH! several types of HVAC systems were investigated. Systems as room-wise electrical heater but also an air-to-air heat pump with a complex air distribution between different zones were realized (see also Calabrese, 2019). Some of the developed models can be found in the library of carnotUIBK.

\section{Simulation}

Within the GUI the simulation can be started. The simulation results can be saved automatically. The actual state of the building model is also saved. This allows to use input parameters also in the post-processing without having a risk of overwriting parameters within the further work. It is also possible (with expert knowledge) to repeat a simulation based on the results.

\section{Post-processing of the Results}

Several simulation studies were performed with this presented model. The automatic post-processing of the tool allows to produce the most relevant figures necessary for a first validation of the results. By accessing the result files (which are MATLAB based data files) user-defined post-processing scripts can be realised.

The following pre-defined functions are available (with automatic captions):

- Temperature, humidity and $\mathrm{CO}_{2}$ plots per zone.

- $\quad$ Sorted temperature plots.

- Energy demand plots for each zone.

- Monthly energy balances for each zone (different losses and gains).

- Heating and cooling power.

- Heat and cool duration curves.

Figure 12 shows for example the heat load duration curve for the presented building with all zones heated to $21.0^{\circ} \mathrm{C}$ and the climate of Innsbruck.



Figure 12: Heat load duration curve.

Figure 13 shows another common plot for the monthly balance of the whole building for the same boundary conditions.

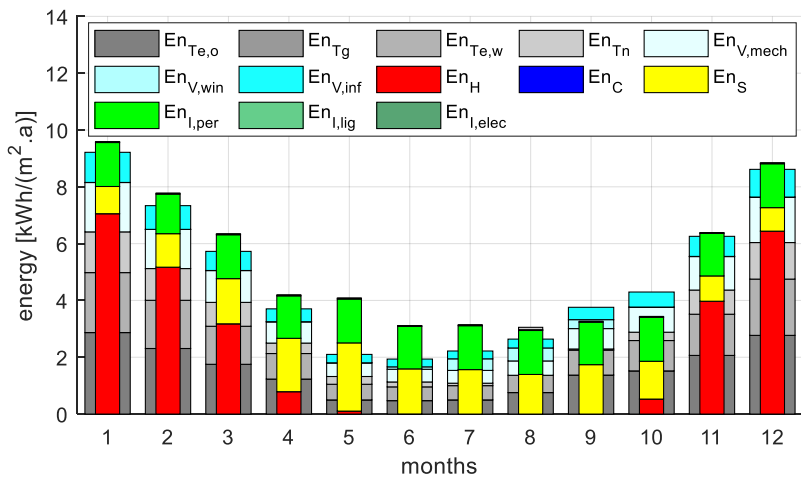

Figure 13: Monthly balance of the whole building.

\section{Conclusion and Outlook}

With the presented carnotUIBK, consisting of a MATLAB object with various sub-objects, a GUI and a Toolbox for Simulink including template, a comprehensive tool for building and HVAC simulation has been developed for the scientific community. To tool 
considers and uses the most recent developments in correlated fields (like B.I.M. integration via gbXML and HiL and co-simulation coupling via BCVTB). With the use of an object-oriented language it can also be used in other fields e.g. use as model in predictive controllers. To publish it as an open source tool with capability to be extended will hopefully help to distribute it and find a community that can provide support and maintenance.

However, the MATLAB building model framework can also be used without Simulink. For this reason it is planned to support also other simulation engines as Modelica and Energy+.

\section{Acknowledgement}

This work is part of the Austrian research project FiTNeS "Fassadenintegrierte modulare Split-Wärmepumpe für Neubau und Sanierung" (2018-21); Förderprogramm Stadt der Zukunft 5. Ausschreibung 2017, FFG Austria, Project number: 867327.

\section{References}

Attia, S., Hensen, J. L.M., Beltrán, L., De Herde, A. (2012). Selection criteria for building performance simulation tools: contrasting architects' and engineers' needs. Journal of Building Performance Simulation, Volume 5, 2012, Issue 3.

Bernal, W., Behl, M., Nghiem, T. X., Mangharam, R. (2013). MLE+: A Tool for Integrated Design and Deployment of Energy Efficient Building Controls. ACM SIGBED Review 10(2):34-34.

Calabrese, T., Ochs, F., Siegele, D. and Dermentzis, G. (2018). Heating with façade integrated heat pumps results of the Austrian FFG project SaLüH!. Proceedings from Advanced Building Skins 2018. Bern (Switzerland), 1-2 October 2018.

CARNOT (2018). CARNOT Toolbox 6.3. Software package.

https://de.mathworks.com/matlabcentral/fileexchange/68 890-carnot-toolbox (29.01.2019).

Crawley, D. B., Hand, J. W., Kummert, M, Griffith, B. T. (2008). Contrasting the capabilities of building energy performance simulation programs. Building and Environment, Volume 43, Issue 4, April 2008, 661-673.

Davies, M.G. (2004). Building Heat Transfer. John Wiley \& Sons. Liverpool (UK).

Dott, R., Haller, M. Y., Ruschenburg, J., Ochs, F., Bony, J. (2013). The Reference Framework for System Simulations of the IEA SHC Task 44 / HPP Annex 38. Part B: Buildings and Space Heat Load. Technical report. Feist, W. (2018). Passive House Planning Package PHPP 9. Software package. Passive House Institute, Darmstadt (Germany).

Feist, W. (1994). Thermische Gebäudesimulation Kritische Prüfung unterschiedlicher Modellansätze. Verlag C.F. Müller. Darmstadt (Germany).

FMI (2019). FMI standard definition. fmi-standard.org (29.01.2019).

Green Building XML (gbXML) Schema, Inc. (2017). gbXML schema. www.gbxml.org/Schema_Current _GreenBuildingXML_gbXML (29.01.2019).
Magni, M., Ochs, F., Geisler-Moroder, D., de Vries, S., Maccarini, A., Calabrese, T. (2019). Comparison of Simulation Results for an Office Building Between Different BES Rools - The Challenge of getting Rid of Modeller Influence and Identifying Reasons for Deviations. Proceedings from Building Simulation 2019. Rome (Italy), 2-4 September 2019.

NREL, ANL, LBNL, ORNL, and PNNL (2018). Open Studio 2.7. Software. www.openstudio.net (29.01.2019). Nytsch-Geusen, C., Inderfurth, A., Kaul, W. (2017). Template based code generation of Modelica building energy simulation models. Proceedings from the 12th International Modelica Conference. Prague (Czech Republic), 15-17 May 2017.

Ochs, F., Siegele, D. and Jähnig, D. (2018). SaLüH!. Research report. FFG Austria. Project number: 850085. 09/2015 - 08/2018.

Rojas, G., Spörk-Dür, M., Venus, D., Greml, A., Krissmer, L., Pfluger, R. (2015). Lüften und Heizen in Passivhäusern in Österreich - Variantenvergleich auf Basis von Behaglichkeit und Nachhaltigkeit. Nachhaltigwirtschaften 36/2015.

Siegele, D., Leonardi, E. (2019). carnotUIBK Toolbox 2.0. Software. www.uibk.ac.at/bauphysik/forschung/ carnotuibk (31.01.2019).

Siegele, D., Ochs, F., Feist, W. (2014). Modelling and Simulation of façade integrated active Components with Matlab/Simulink. Proceedings from BauSIM 2014: Human-centred building(s). Aachen (Germany), 22-24 September 2014.

Siegele, D. and Ochs, F. (2018). Einsatz von Enthalpieübertragern im Mehrgeschosswohnbau in kalten Klimaten. Proceedings from BauSIM 2018. Karlsruhe (Germany), 26-28 September 2018.

Trimble Inc. (2018). Sketchup 2017. Software. www.sketchup.com (29.01.2019).

Vogelsang, S., Fechner, H., Nicolai, A. (2013). Delphin 6 Material Specification. Technical Report. Technical University of Dresden (Germany).

Wetter, M. (2010). Co-simulation of building energy and control systems with the Building Controls Virtual Test Bed. Journal of Building Performance Simulation 4(3), 185-203 